\title{
GPON Service Level Agreement based Dynamic Bandwidth Assignment Protocol
}

\author{
Ching-Hung Chang, P. Kourtessis and J. M. Senior
}

A dynamic bandwidth assignment protocol is proposed that demonstrates impartial and highly efficient bandwidth arrangement for gigabit-capable passive optical networks. In particular the novel algorithm automatically modifies the assigned bandwidth per optical network unit to support quality of service and service level agreement according to maximum network capacity and users' queuing status. Network simulation results have demonstrated superior mean packet delay performance achieving a tenfold reduction of packet delay at high network load when compared to other dynamic bandwidth assignment algorithms.

Introduction: Time division multiplexing-passive optical networks have been adopted to provide a cost-effective solution for the implementation of point to multipoint (P2MP) future broadband access networks [1]. gigabit-capable passive optical networks (GPONs) in particular support the development of full service access networks (FSANs) by providing multiple network rates downstream and upstream, and guaranteed quality of service (QoS) over distances of up to $60 \mathrm{~km}[2]$.

According to the standard [2], the optical line terminal (OLT) assigns available time slots to each optical network unit (ONU) for transmission, by means of 
grant packets, according to the report packets send by each ONU to notify the OLT about their buffer queuing status. To avoid the accumulation of "waiting time" associated with the report and grant packets polling times, an interleaved polling scheme with adaptive cycle time (IPACT) [3] has been proposed for ethernet-PONs (EPONs) to overlap multiple polling requests in time. Although intended for EPON applications the interleaved polling scheme can be directly applied to allocate bandwidth in GPON architectures, being also of tree topology, by readjusting the time to transmit the grant packets from the OLT to each ONU and redesigning the frame format.

Consequently IPACT can utilize bandwidth more efficiently than constant time-slot PONs [3], and therefore it has been used to develop other EPON dynamic bandwidth assignment (DBA) algorithms, some examples of which are the dynamic credit distribution [4], guaranteed minimum bandwidth [5], two-layer bandwidth allocation [6] and Intra-ONU bandwidth allocation schemes [7]. What all these approaches have in common is that ONUs are assigned to a single service level and same aggregate data rates. However, the GPON standard [2] defines multiple data rates and the internet service providers (ISPs) in practice, assign multi-service levels according to customers' requirements.

This paper proposes a novel DBA scheme for GPON which achieves QoS at three different types of service levels and diverse network throughputs. Initially it dynamically assigns to each ONU a guaranteed minimum bandwidth from the overall network capacity to satisfy their basic service requirements. 
Consequently, the OLT will apportion any unused bandwidth to ONUs according to their buffer queuing status. Therefore, following probable variations in network capacity, the OLT will be capable of readjusting the guaranteed minimum and unused bandwidths among ONUs to comply with subscriber contracts.

Proposed algorithm: The OLT is designed to provide ONUs with three service levels, denoted by $t$, at different weights $W_{t}$ to represent the priority of accessing the network. As a result service level one for example will acquire the lowest weight which will reflect the amount of time slots it will occupy in one polling cycle. To simulate practical network conditions, these weights are selected in order to comply with the NTT VDSL service plans corresponding to 50,70 and $100 \mathrm{Mbit} / \mathrm{s}$ for service levels with weights 2,3 , and 4 respectively [8]. The transmission time-slots for each user are assigned in two stages. In the first stage, a guaranteed minimum bandwidth, $B_{\text {min }}^{t}$, is assigned to each ONU according to its service level. In particular to provide diverse service levels and being able to adapt to variable data rates, $B_{\min }^{t}$ should be defined as the supplement of two parameters; a basic bandwidth, $B_{\text {basic }}$, and an extra guaranteed bandwidth, $B_{e x}^{t}$. Henceforth, the guaranteed minimum bandwidth for service level $t$ will be given by:

$$
B_{\min }^{t}=B_{\text {basic }}+B_{e x}^{t}
$$

In eq. 1 , the basic bandwidth, $B_{\text {basic }}$, describes a constant bandwidth available to all ONUs independently of service level. In order for the OLT to dynamically assign more bandwidth to higher service level ONUs at variable data rates, 
the extra bandwidth parameter, $B_{e x}^{t}$, is included in the calculation of $B_{\min }^{t}$. For service level $t$, the extra bandwidth, $B_{e x}^{t}$, is assigned by considering the maximum network capacity $B_{\text {total }}$ and is therefore given by:

$$
B_{e x}^{t}=\left(B_{\text {total }}-k \times B_{\text {basic }}\right) \frac{W_{t}}{\sum_{t=1}^{3} W_{t} N_{t}}
$$

where $k$ is the number of ONUs comprising the network and $N_{t}$ is the number of ONUs subscribed to service level $t$.

In the probability that in each polling cycle only a fraction of ONUs use their entire guaranteed minimum bandwidth, the algorithm in the second stage will proportionally assign the unused bandwidth to the ONUs whose requirement exceed $B_{\text {min }}^{t}$, as calculated before. Therefore for $\mathrm{ONU}_{i}$ the extra assigned

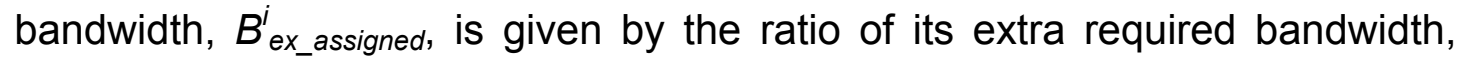

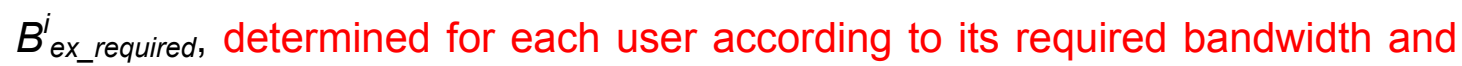
relative $B_{\text {min }}^{t}$, over the total extra required bandwidth multiplied by the unused bandwidth following:

$$
B_{\text {ex_assigned }}^{i}=\sum B_{\text {unused }} \times \frac{B_{\text {ex_ requried }}^{i}}{\sum B_{\text {ex__required }}}
$$

The unused bandwidth for $\mathrm{ONU}_{i}$ from stage one can be calculated by subtracting the corresponding queuing length $Q_{i}$, representing the bandwidth requirement, from the total minimum guaranteed bandwidth.

In summary, the maximum allocated bandwidth $B_{\text {max_allocated }}$ for $\mathrm{ONU}_{i}$ will be equal to the summation of $B_{\text {min }}^{t}$ from stage one and $B_{\text {ex_assigned }}$ from stage two. 
Otherwise if ONU's request for bandwidth is smaller than the total, $B_{\text {max_allocated }}$ will be equal to $Q_{i}$ as given below:

$$
B_{\text {max_allocated }}^{i}=\min \left(\begin{array}{l}
B_{\min }^{t}+B_{\text {ex_assigned }}^{i} \\
Q_{i}
\end{array}\right)
$$

The new algorithm which is designed to grant network users with diverse service levels improved performance is called the dynamic minimum bandwidth (DMB) scheme.

Network modeling and simulation results: To evaluate the performance of the proposed scheme, a GPON network was devised in OPNET modeler comprising a single OLT and 16 ONUs with varying weights $\left(1^{*} W_{3}+5^{*} W_{2}+\right.$ $\left.10^{*} W_{1}\right)$ to represent different service levels. Since the fundamental differentiation in implementing EPON and GPON DBA algorithms is in the frame format, a simple GPON packet header was modeled to simulate the upstream transmission of $1 \mathrm{Gbit} / \mathrm{s}$ for link lengths of up to $25 \mathrm{~km}$ [2]. In addition, a $2 \mathrm{~ms}$ maximum cycle period and $1 \mu \mathrm{s}$ guard time were chosen to establish data transfer between the OLT and ONUs in order to offer a platform for comparison with published algorithms [4-7]. Network traffic was generated based on a self-similar model with typical Hurst parameter of 0.8 , maximum ONU channel capacity of $100 \mathrm{Mbit} / \mathrm{s}$ and basic bandwidth of $34 \mathrm{Mbit} / \mathrm{s}$ to effectively simulate the requirement in bandwidth of representative network services. 
The simulated channel throughput versus network load characteristics for IPACT and the proposed DMB scheme are shown in Fig. 1. To be able to provide a valid evaluation of the proposed algorithm's performance against IPACT, taking into consideration that the latter was intended for EPON applications, preliminary work investigated the successful adaptation of IPACT to the GPON model. It may be observed that IPACT can be equally applied to EPON and GPON architectures establishing its validity as a reference for assessing the proposed scheme. In addition, the two algorithms exhibit similar performance when modeled as GPON as far as the achieved network throughput is concerned indicating identical bandwidth utilisation.

Fig. 2, however, which displays the simulated mean packet delay against network load demonstrates a diversion in performance between the two algorithms. In this case the DMB exhibits around a factor of 10 less packet delay in comparison with IPACT under high network load, suggesting that the proposed algorithm is much more efficient in utilizing the limited network capacity. This is due to the fact that when the maximum network capacity has been reached, the ONUs with higher requirement in bandwidth are allocated most of the available network bandwidth in priority.

Finally, Fig. 3 shows the simulated network packet delay response for the assigned service levels. It becomes apparent from the plot that when each ONU's offered load is greater than half its maximum capacity, total user requirement in bandwidth exceed the network capacity. Therefore for increasing values of network load, the OLT will proportionally allocate most of 
the unused bandwidth among the higher service level ONUs, resulting to less packet delay for extended load figures, given by $0.54,0.61$ and 0.74 for service levels 1,2 , and 3 respectively.

Conclusion: This paper has demonstrated a novel DBA algorithm exhibiting impartial and highly efficient bandwidth arrangement for TDM-GPONs. In particular the maximum bandwidth allocated per ONU is dynamically assigned, based on network capacity, subscriber service levels and queuing status aiming to assign more bandwidth to higher service level subscribers and consequently to reduce their packet delay compared to their low service counterparts. Contrasting the proposed scheme with alternative reported EPON algorithms, simulation results have demonstrated reduced mean packet delay accomplishing up to a tenfold decrease at high network load. In addition, the demonstrated network performance in packet delay of high service level users is sustained for increased traffic demonstrating network integrity and QoS according to subscriber service levels.

\section{References}

$1 \quad$ X.-Z. Qiu, P. Ossieur, J. Bauwelinck, Y. Yi, D. Verhulst, J. Vandewege, B. DeVos, and P. Solina, "Development of GPON Upstream PhysicalMedia-Dependent Prototypes," Journal of Lightwave Technology, vol. 22, pp. 2498-2508, 2004.

2 ITU-T Recommendation. G.984.2, "Gigabit-capable passive optical networks (GPON): Physcal media dependent (PMD) layer specification," 2003.

3 G. Kramer, B. Mukherjee, and G. Pesavento, "IPACT a dynamic protocol for an Ethernet PON (EPON)," Communications Magazine, IEEE, vol. 40, pp. 74-80, Feb 2002. 
$4 \quad$ H. Miyoshi, T. Inoue, and K. Yamashita, "QoS-aware dynamic bandwidth allocation scheme in Gigabit-Ethernet passive optical networks," IEEE International Conference on Communications, vol. 1, pp. 90-94, 2004.

$5 \quad$ D. Nowak, P. Perry, and J. Murphy, "Bandwidth Scheduling Techniques for Differentiated Services Support in Ethernet Passive Optical Networks," Proc. of 9th International Workshop on Component Oriented Programming (WCOP), in conjunction with 18th European Conference on Object-Oriented Programming (ECOOP), June 2004.

6 J. Xie, S. Jiang, and Y. Jiang, "A dynamic bandwidth allocation scheme for differentiated services in EPONs," Communications Magazine, IEEE, vol. 42, pp. S32-S39, Aug 2004.

7 N. Ghani, A. Shami, C. Assi, and M. Y. A. Raja, "Intra-ONU bandwidth scheduling in Ethernet passive optical networks," Communications Letters, IEEE, vol. 8, pp. 683-685, 2004.

$8 \quad$ NTT, "NTT VDSL service plan," http://www.asist.co.jp/jensspinnet/bflets.html, Feb 2006.

\section{Authors' affiliations:}

Optical Networks Group, Science and Technology Research Institute (STRI), University of Hertfordshire, College Lane Campus, Hatfield, Herts AL10 9AB, UK.

\section{E-mail addresses:}

P.Kourtessis@herts.ac.uk

C.H.Chang@herts.ac.uk

\section{Figure captions:}

Fig. 1 Channel throughput for IPACT and Dynamic Minimum Bandwidth in GPON

Fig. 2 Mean packet delay for IPACT and Dynamic Minimum Bandwidth in GPON

Fig. 3 Packet delay for three service levels in Dynamic Minimum Bandwidth in GPON

Figure 1 


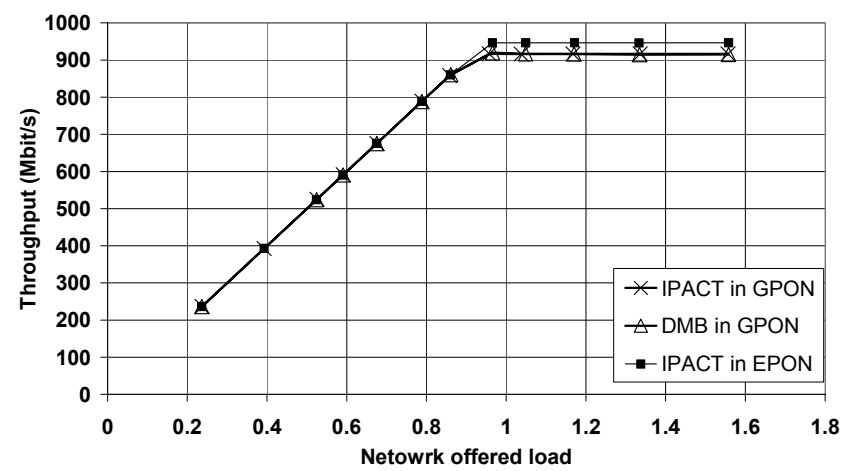

Figure 2

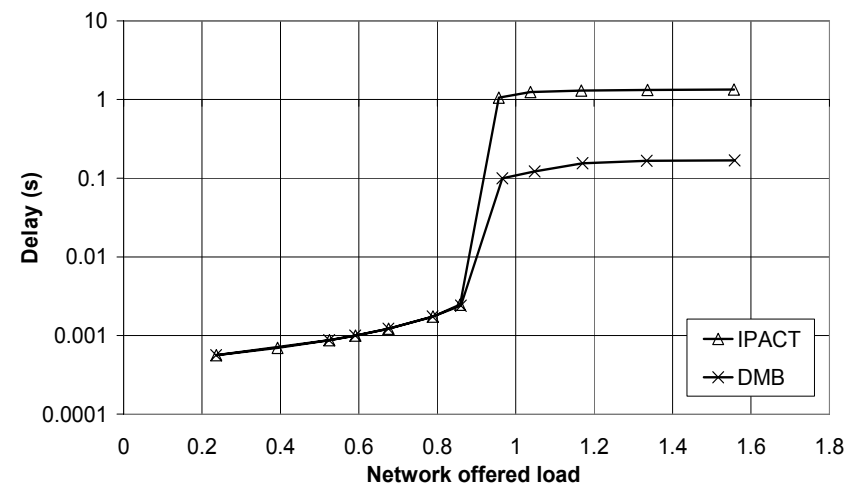

Figure 3

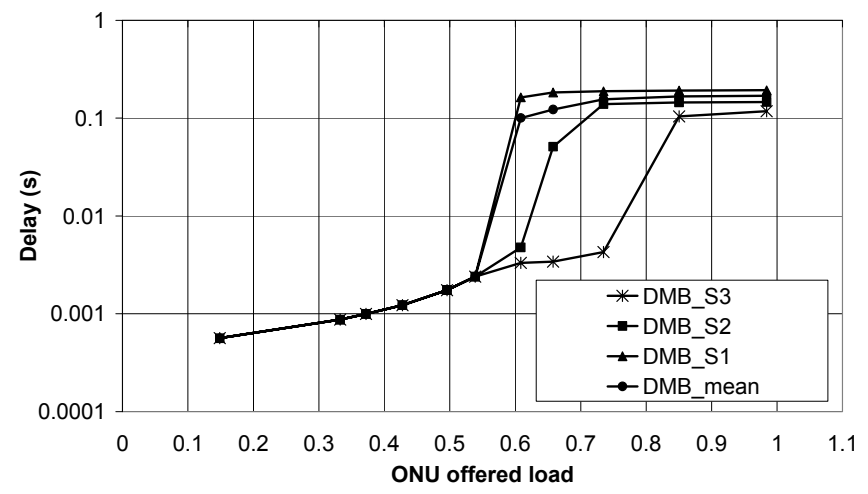

